\section{Localization of acoustical modes due to the electron-phonon interaction within a two- dimensional electron gas}

To cite this article: V A Kochelap and O Gulseren 1993 J. Phys.: Condens. Matter 5589

View the article online for updates and enhancements.

\section{Related content \\ The physics of quantum well structures M J Kelly and R J Nicholas \\ One-dimensional Fermi liquids \\ Electronic transport in low-dimensional structures \\ J J Harris, J A Pals and R Woltjer}

\section{Recent citations}

$$
\begin{aligned}
& \text { - RECENT DEVELOPMENTS ON } \\
& \frac{\text { ELECTRON-PHONON INTERACTIONS }}{\text { IN STRUCTURES FOR ELECTRONIC }} \\
& \frac{\text { AND OPTOELECTRONIC DEVICES }}{\text { M. DUTTA et al }} \\
& \text { - Renormalization of acoustic phonon } \\
& \frac{\text { spectra and rudiments of the Peierls }}{\text { transition in free-standing quantum wires }} \\
& \text { K. W. Kim et al } \\
& \text { - Capillary phenomena and macroscopic } \\
& \frac{\text { dynamics of complex two-dimensional }}{\text { defects in crystals }} \\
& \text { Yuriy A. Kosevich }
\end{aligned}
$$

\section{IOP ebooks}

Bringing you innovative digital publishing with leading voices to create your essential collection of books in STEM research. Start exploring the collection - download the first chapter of every title for free. 


\title{
Localization of acoustical modes due to the electron-phonon interaction within a two-dimensional electron gas
}

\author{
V A Kochelapł and $O$ Gülseren \\ Department of Physics, Bilkent University, Bilkent 06533, Ankara, Turkey
}

Received 10 June 1992, in final form 26 November 1992

\begin{abstract}
We study the localization of acoustical modes within a two-dimensional electron gas (2DEG) due to only the electron-phonon interaction. The localized modes split out from the bulk phonons even at uniform lattice parameters, when the $2 D E G$ is created by means of modulation doping, for example under $\delta$-doping. The effect is more pronounced when the wave vector $q$ of the modes increases and is maximum at $q=2 k_{\mathrm{F}}\left(k_{\mathrm{F}}\right.$ is the Fermi wave vector). In the case of several electron sheets the additional features of the localization effect appear.
\end{abstract}

\section{Introduction}

One of the current topics of the semiconductor physics is the localization or confinement of phonons in semiconductor heterostructures [1-4]. This phenomenon is interesting because of its fundamental aspects, as well as applications. In particular, the phonon localization affects the electron transport, which is the main object of applications of the heterostructures. It is well known that phonon localization in heterostructures is due to the different lattice characteristics of the semiconductor compounds forming the heterostructure (various lattice constants, lattice forces, symmetry, etc). On the other hand, the existence of free carriers in these layers is not considered as the main reason for the localization effect.

In this paper we predict and study phonon localization (confinement) originating from the electron-phonon interaction. We show that localization of acoustical modes appears due to only the electron-phonon interaction if there is an electron gas sheet ( $3 \mathrm{D}$ or $2 \mathrm{D}$ electron layer). The lattice characteristics are uniform before inserting the electron sheet, therefore this effect exists even at uniform lattice characteristics except the modifications due to the electron-phonon interaction. Such a physical situation and the electron layers can be realized by modulation doping, for example under $\delta$-doping [5-7].

The physical basis of this localization effect mechanism is the same as the physics of renormalization of acoustical vibrations by the electrons in bulk materials, a subject which has been studied theoretically and experimentally before [8]. It can be explained qualitatively as follows. An acoustical wave creates a potential for electrons. Electrons having high velocities follow the acoustical wave. Hence they are

† Permanent address: Theoretical Physics Department, Institute of Semiconductors, Academy of Science of Ukraine, Kiev-252650, Ukraine. 
redistributed in space. Non-uniform redistribution of the electrons leads to spacedependent electronic forces which act on the lattice, expanding the lattice in some regions while compressing it in others. Renormalization of the phonon spectrum arises as a result of such a self-consistent interaction. The sound velocity is always decreased by this renormalization, which means a reduction of the elastic modulus and a softening of the lattice. In addition to that, it gives rise to a set of other effects such as the features of the spectrum at the phonon wave vector $q=2 k_{\mathrm{F}}\left(k_{\mathrm{F}}\right.$ is the Fermi electron wave vector) and the displaying of the electron system symmetry by the renormalized acoustical spectrum.

In the case when electrons are confined within some layer, the above-mentioned mechanism causes a slight decrease of the elastic modulus of the layer. From the theory of elastic waves [1-3] it is known that an embedded layer characterized by a decreased elastic modulus always splits the bulk acoustical spectrum into bulklike modes and localized modes. The latter are confined to lie within or near the embedded layer, and propagate along the layer. Therefore we can expect that the electron-phonon interaction under the localization of electrons within the electron sheet would lead to the phonon localization effect. The aim of this study is to show the existence of this phenomenon.

\section{Model and equations}

We will describe the long-range acoustical vibrations of lattice by the equation for sound waves [9]

$$
\rho \partial^{2} u_{i} / \partial t^{2}=\partial \sigma_{i k} / \partial x_{k}
$$

where $u_{i}$ are components of the displacement vector $(u)$ of the medium, $\rho$ is its density, $\sigma_{i k}$ is the stress tensor, $t$ and $x_{k}$ are time and space coordinates, respectively. In this paper we use the Einstein summation convention where the repeated indices are summed over. For simplicity, we consider the isotropic elastic medium. Then, the contribution of elastic lattice forces to the stress tensor $\sigma_{i k}$ is [9]

$$
\sigma_{i k}^{(L)}=\left(\lambda+\frac{2}{3} \mu\right) u_{l l} \delta_{i k}+2 \mu\left(u_{i k}-\frac{1}{3} \delta_{i k} u_{l l}\right)
$$

where $\lambda$ and $\mu$ are Lamé coefficients, and $u_{i k}$ is the strain tensor. We assume also that the electrons are characterized by the isotropic energy law. Then the electronphonon interaction can be described by only one constant of the deformation potential $b[10]$ and the contribution of the electrons to the strain tensor $\sigma_{i k}$ is

$$
\sigma_{i k}^{(E)}=b n \delta_{i k}
$$

where $n$ is the electron concentration. This term can be derived from the free energy of the system [11] which includes the electron-phonon interaction in the form $b n \nabla u$.

Inserting equations (2) and (3) into equation (1), the equation for the displacement $u_{i}$ is obtained

$$
\rho \partial^{2} u_{i} / \partial t^{2}=(\lambda+\mu) \partial u_{l l} / \partial x_{i}+\mu \nabla^{2} u_{i}+b \partial n / \partial x_{i}
$$


We assume that the electrons are confined in a sheet of thickness $d$ by a corresponding potential (for example, by the electrostatic potential of the positive charge of donor sheet). The phonon wave vector $q$ is restricted such that the characteristic decay length of modes outside the sheet $\kappa_{\mathrm{ch}}^{-1}(q)$ is much larger than the layer thickness $d$. The criterion is

$$
\kappa_{\mathrm{ch}}(q) d \ll 1 \text {. }
$$

In such a case it is possible to consider the electrons as confined in a plane (for example, in the plane $z=0$, where $z$ is the direction perpendicular to the plane). Hence the electron concentration can be written as

$$
n(r, t)=n_{s}(x, y) \delta(z)
$$

where $n_{\mathrm{s}}$ is the 'surface' concentration of the electrons. However, electron motion can have a $2 \mathrm{D}$ and $3 \mathrm{D}$ character depending on the number of electron subbands. We consider that the electrons follow adiabatically the vibration of lattice and are redistributed in the potential of the acoustic wave according to the physical picture presented in the introduction. The inequality $\bar{\epsilon} \gg \hbar \omega$, which is a necessary condition of this adiabatic approximation, always holds for the semiconducurs ( $\bar{\epsilon}$ is the characteristic electron energy, $\omega$ is the phonon frequency, see appendix). The potential induced by the acoustic wave is

$$
h(r)=b u_{l l}-e \varphi .
$$

Here the term $b u_{l l}$ describes the change of the bottom of the electron zone due to the deformation of the lattice, and $\varphi$ is the electrostatic potential arising from the non-uniform redistribution of the electrons in space and it is governed by the Poisson equation

$$
\nabla^{2} \varphi=\left(4 \pi e / \epsilon_{0}\right) \delta n_{\mathrm{s}}(x, y) \delta(z)
$$

where $\epsilon_{0}$ is the dielectric constant of crystal.

We assume the dependence of all the variables on the plane coordinates $(x, y)$ has the form

$$
u_{i}, \varphi, h, \delta n_{s} \propto \mathrm{e}^{\mathrm{i} q \cdot r \|}
$$

where $q$ lies in the plane of the electron sheet and $r_{\|}=r_{\|}(x, y)$. Any change in the electron concentration $\delta n$ can be calculated by using the perturbation theory for the density operator (see appendix):

$$
\delta n_{\mathrm{s}}(x, y)=h(x, y, z=0) P(q, T)
$$

Here

$$
P(q, \Gamma)=-\sum_{n, k} \frac{f_{0}\left(\epsilon_{n, k+q}\right)-f_{0}\left(\epsilon_{n, k}\right)}{\epsilon_{n, k+q}-\epsilon_{n, k}+\mathrm{i} \delta}
$$

is the polarization of the electron subsystem. $\epsilon_{n, k}$ is the electron energy for the $n$th subband, $k$ is the wave vector of the electrons describing their motion in the plane of the electron sheet, and $f_{0}\left(\epsilon_{n, k}\right)$ is the Fermi distribution function. If the number of subbands is large, the electron motion within the sheet is almost three-dimensional. If the number of subbands is small, the polarization $P(q, T)$ corresponds to the electron gas with reduced dimensionality.

The set of relationships (4), (6-8), (10), (11) is sufficient to consider the acoustical modes localized near the electron layer. 


\section{Solutions for localized modes and their analysis}

It is easy to prove using equation (4) that only longitudinal acoustical waves interact with electrons in our model of the isotropic energy spectrum of the electrons. Therefore it is convenient to consider the equation for relative volume change $\boldsymbol{\nabla} \cdot \boldsymbol{u} \equiv u_{l}$ instead of several components of the displacement of the lattice. From equation (4) we find the equation:

$$
\rho \frac{\partial^{2}}{\partial t^{2}}(\nabla \cdot u)-(\lambda+2 \mu) \nabla^{2}(\nabla \cdot u)=b \nabla^{2} n .
$$

Now, by taking into account the relationships (6) and (9), we can write the system of equations in the following simple form for the region outside the plane $z=0$ :

$$
\begin{array}{ll}
{\left[\frac{\mathrm{d}^{2}}{\mathrm{~d} z^{2}}-\left(q^{2}-\frac{\omega^{2}}{c_{\mathrm{L}}^{2}}\right)\right] u_{l l}=0} & c_{\mathrm{L}}^{2}=\frac{\lambda+2 \mu}{\rho} \\
\left(\frac{\mathrm{d}^{2}}{\mathrm{~d} z^{2}}-q^{2}\right) \phi=0 & \phi=-e \varphi
\end{array}
$$

Here $\omega$ is the frequency of the determined waves, $c_{\mathrm{L}}$ is the velocity of the longitudinal acoustical phonons for the system without electrons. The solutions of equations (13) outside the sheet (at $z \neq 0$ ) should be matched at $z=0$ by the following conditions:

$$
\begin{aligned}
& \frac{\mathrm{d} u_{l l}}{\mathrm{~d} z}(+0)-\frac{\mathrm{d} u_{l l}}{\mathrm{~d} z}(-0)=\frac{b}{\lambda+2 \mu} q^{2} \delta n_{\mathrm{s}} \\
& \frac{\mathrm{d} \phi}{\mathrm{d} z}(+0)-\frac{\mathrm{d} \phi}{\mathrm{d} z}(-0)=-\frac{4 \pi e^{2}}{\epsilon_{0}} \delta n_{\mathrm{s}} \\
& \delta n_{\mathrm{s}}=-P(q, T)\left(b u_{n}+\phi\right)_{z=0} \\
& \phi(+0)=\phi(-0) \quad u_{l l}(+0)=u_{l l}(-0)
\end{aligned}
$$

and satisfy the boundary conditions far away from the sheet:

$$
u_{l l}, \phi \longrightarrow 0 \quad z \longrightarrow \pm \infty .
$$

In equations (14), $u_{l l}( \pm 0)$ and $\phi( \pm 0)$ are the solutions at the left- and right-hand side of the sheet in the limit $z \rightarrow \pm 0$. The conditions (16) mean that we look for solutions localized near the electron sheet.

The solutions of equations (13) can be written for both $u_{31}$ and $\phi$ from equation (15) by using continuity conditions as

$$
u_{l l}=A \mathrm{e}^{-\kappa|z|} \quad \kappa=\sqrt{q^{2}-\omega^{2} / c_{\mathrm{L}}^{2}} \quad \phi=B \mathrm{e}^{-q|z|} .
$$

Inserting these solutions into equation (14) gives the relationship between the magnitudes of the acoustic wave and the vibrations of the electrostatic potential:

$$
B=-A b\left[4 \pi e^{2} P(q, T) / \epsilon_{0}\right] q^{3} /\left[2 q+4 \pi e^{2} P(q, T) / \epsilon_{0}\right] .
$$


The expression for $\kappa$ is

$$
\kappa=\sqrt{q^{2}-\omega^{2} / c_{\mathrm{L}}^{2}}=\left[b^{2} P(q, T) /(\lambda+2 \mu)\right]\left[q^{3} /\left(2 q+4 \pi e^{2} P(q, T) / \epsilon_{0}\right)\right] .
$$

The right-hand side of equation (19) is always positive. This means that the solutions decay exponentially far away from the layer. The same expression gives the dispersion relation for the localized acoustical modes

$\omega^{2}=q^{2} c_{L}^{2} \llbracket 1-\left\{\left[b^{2} P(q, T) /(\lambda+2 \mu)\right] q^{2} /\left(2 q+4 \pi e^{2} P(q, T) / \epsilon_{0}\right)\right\}^{2} \rrbracket$.

One can see from (20) that the frequencies of the localized phonons are always less than the frequencies of the bulk one. That is consistent with the physical picture described in the introduction. The splitting value of the frequencies depends on the fourth power of the constant coupling $b$. The difference between the bulk phonons and the localized one also grows with increasing $q$ : the degree of the localization becomes larger as is seen from relation (19), and the dispersion relation falls off from the linear behaviour.

The term $4 \pi e^{2} P(q, T) / \epsilon_{0}$ in the denominator of expressions (18)-(20), evidently, describes the screening effect of the electron charge which is redistributed in the electron sheet. It is convenient to introduce the characteristic wave vector $q_{s c}$ by the equality

$$
q_{\mathrm{sc}}=\left(2 \pi e^{2} / \epsilon_{0}\right) P\left(q_{\mathrm{sc}}\right) .
$$

Since the function $P(\boldsymbol{q}, T)$ decreases with increasing $q$ for both 3D and 2D systems the equation (21) has only one solution for $q_{\mathrm{sc}}$. In the case of

$$
q<q_{\mathrm{sc}}
$$

the total potential $h(r)$ induced by the acoustical wave is small as follows from (7) and (17), because the change of the bottom of the conduction band $b(\nabla \cdot u)$ and the electrostatic energy $-e \varphi$ compensate each other. In this limiting case $\left(q \ll q_{\mathrm{sc}}\right)$ the dispersion relation takes a simple form

$$
\omega^{2}=q^{2} c_{\mathrm{L}}^{2}\left\{1-\left[b^{2} \epsilon_{0} q^{2} / 4 \pi e^{2}(\lambda+2 \mu)\right]^{2}\right\}
$$

and does not depend on parameters of the electron band, quantization into the sheet, temperature, etc. Of course, this simple expression is valid under certain conditions like those given by (5) and (22). In this limit, the decay length $\kappa^{-1}$ of the acoustic mode outside the sheet is proportional to $q^{-3}$.

In contrast to (22), in the case

$$
q>q_{\mathrm{sc}}
$$

the screening is not essential. Equation (19) implies that we can neglect the term $-e \varphi$ in (7) and the dispersion relation takes the form (for $q \gg q_{\mathrm{sc}}$ )

$$
\omega^{2}=q^{2} c_{\mathrm{L}}^{2} \llbracket 1-\left\{\left[b^{2} P(q, T) / 2(\lambda+2 \mu)\right] q\right\}^{2} \rrbracket .
$$

In this case, the magnitude of the mode outside the electron sheet decays like $\kappa^{-1} \sim q^{-2}$. For estimating the inequalities (5), (22), (24) and discussing the dispersion 
relation, it is necessary to calculate $P(g, T)$. At the low-temperature limit we can find from equation (11)

$$
\begin{aligned}
& P(q)=\sum_{n} P_{n}(q) \quad T \rightarrow 0 \\
& P_{n}(q)=\left(m^{*} / \pi \hbar^{2}\right)\left\{1-\left(2 k_{\mathrm{F}}^{(n)} / q\right)\left[\left(q / 2 k_{\mathrm{F}}^{(n)}\right)^{2}-1\right]^{1 / 2} \Theta\left[\left(q / 2 k_{\mathrm{F}}^{(n)}\right)-1\right]\right\}
\end{aligned}
$$

where the summation is over all occupied subbands, $k_{\mathrm{F}}^{(n)}$ is the Fermi wave vector of the electrons in the $n$th subband $\left(\hbar^{2}\left(k_{F}^{(n)}\right)^{2} / 2 m^{*}=\epsilon_{F}-\epsilon_{n, 0}, \epsilon_{F}\right.$ is the Fermi energy), $\Theta$ is the Heaviside step function [12]. In this limit, the relationship of the Fermi energy and the total surface concentration of electrons is given by

$$
n_{\mathrm{s}}=\frac{m^{*}}{\pi \hbar^{2}} \sum_{n}\left(\epsilon_{\mathrm{F}}-\epsilon_{n, 0}\right)
$$

where the summation is calculated over the occupied subbands.

In the limiting case of high temperature $T$ the polarization is obtained as

$$
P(\boldsymbol{q}, T)=n_{\mathrm{s}} / T \text {. }
$$

As seen from (26)-(28), the parameter $q_{\text {sc }}$ characterizing the screening and also the localization effect increases with decreasing temperature. Because of this we will consider the case of low temperature in detail. The following analysis will clarify the physical picture and describes the actual situation $q_{\mathrm{sc}}<q$ better. The value $q_{\mathrm{sc}}$ is always small comparable with $k_{\mathrm{F}}^{(n)}$ for semiconductors with large dielectric constant $\epsilon_{0}$ (for example, IV-VI compounds):

$$
q_{\mathrm{sc}}<k_{\mathrm{F}}^{(n)} \text {. }
$$

This means that in the region $q \sim k_{\mathrm{F}}$ where the localization effect is more pronounced, the screening does not suppress the effect. In general, for semiconductors with modest $\epsilon_{0}$, inequality (29) can also hold. It follows from (26) that the maximum value of $q_{\mathrm{sc}}$ is of the order of the inverse Bohr radius $a_{\mathrm{B}}$ for semiconductors. In fact, let the electron sheet be created by means of doping. It is necessary to dope the semiconductor up to concentrations such that $n_{\mathrm{s}} a_{\mathrm{B}}^{2}>1$ to obtain free carriers and conductivity $[6,13]$. But this inequality is equivalent to the criterion (29). When (29) holds, we can consider the case $q \sim k_{\mathrm{F}}$ and (24) would be valid. The decay length of the acoustic wave outside the electron sheet $\kappa^{-1}=2(\lambda+2 \mu) / b^{2} P(q, T) q^{2}$ is much larger than the wavelength $2 \pi / q$ even for $q \sim k_{\mathrm{F}}$ and $q \sim 1 / d$ for actual semiconductor parameters (see estimates below). Since the inequalities (5), (24) and (29) are compatible, (i.e. the expression for the dispersion relation (25) holds in the region $q \sim k_{\mathrm{F}}$ ), the electrostatic potential can be neglected and the splitting of the localized acoustical mode is maximal. At $q>2 k_{\mathrm{F}}$ the magnitude of $P(q) q$ is proportional to $q^{-1}$, so the splitting decreases with increasing $q$.

In order to estimate the magnitude of the localization effect, we use the following parameter values relevant to most common semiconductors of general interest: $\lambda+2 \mu=10^{12} \mathrm{~g} \mathrm{~cm}^{-1} \mathrm{~s}^{-2}, b=15 \mathrm{eV}, m=0.5 m_{0}$ (p-type material), $\epsilon_{0}=15$. Then 


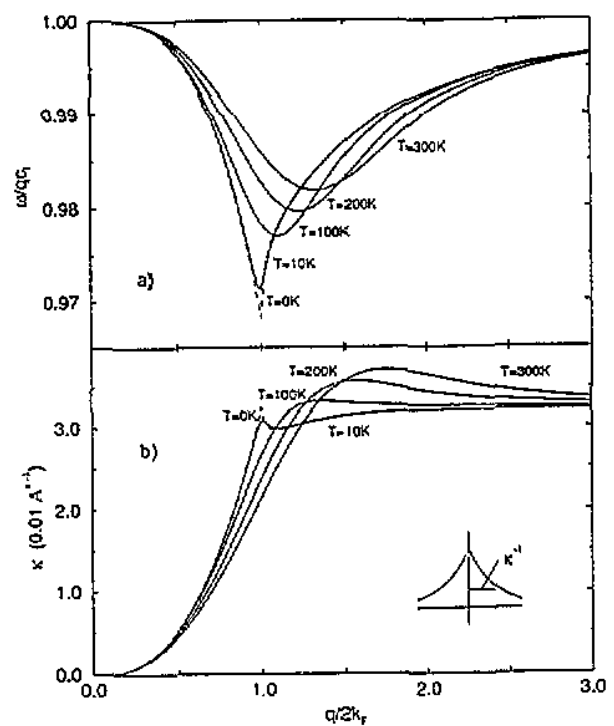

Figure 1. Characteristics of localized modes as a function of wave vector $q$ for a single electron sheet at different temperatures: (a) phase velocity $\omega / q c_{L},(b)$ inverse decay length $\kappa$. The maximum of the splitting in frequency is on the order of $10^{11} \mathrm{~s}^{-1}$. The inset shows the form of the solution.

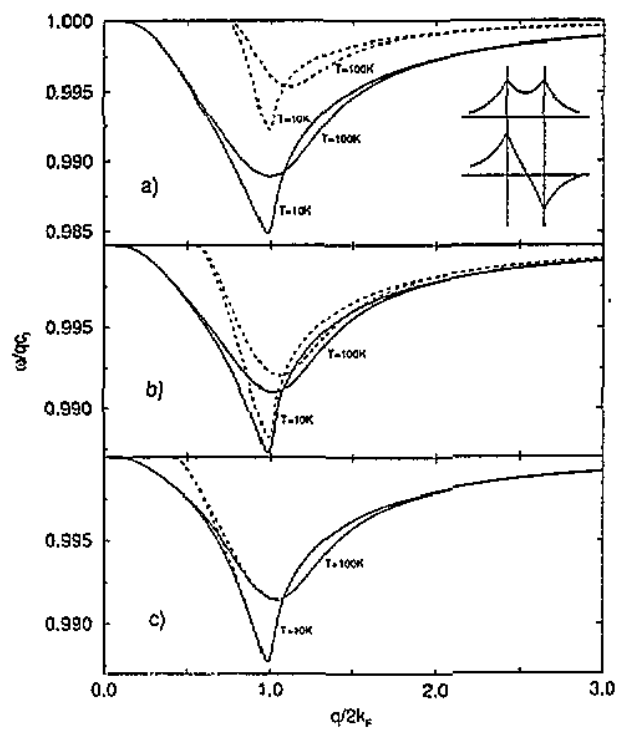

Figure 2. Phase velocity $\omega / q c_{\mathrm{L}}$ as a function of wave vector $q$ for two identical electron sheets at two different temperatures. Interlayer distance $2 L$ is equal to (a) $100 \AA$, (b) $200 \AA$ and (c) $400 \AA$. While antisymmetric solutions are shown by dashed lines, symmetric solutions are shown by solid lines. The inset shows the forth of symmetric and antisymmetric solutions.

for a typical electron concentration for a $\delta$-doping layer $n=6.7 \times 10^{12} \mathrm{~cm}^{-2}[5,6]$ $\left(k_{\mathrm{F}}=6.488 \times 10^{6} \mathrm{~cm}^{-1}\right.$, if we assume a single subband), we find the maximum of the splitting of the localized modes $\left(\omega_{\text {bulk }}-\omega_{\text {conf }}\right) / \omega_{\text {bulk }}=0.03$. The modes are localized inside a region of thickness $80 \AA$. For semiconductors with a large dielectric constant (IV-VI compounds) the splitting increases by as much as threefold. Quantitative behaviour of $\omega(q)$ for the localized acoustical mode is shown in figure 1.

\section{Localization of acoustical modes for the cuse of two electron sheets}

In previous sections we considered the localization effect for the acoustical modes caused by a single electron sheet. We have shown that the characteristic scale of the decaying of the waves in space outside the electron sheet can be considerable. It is known that not only heterostructures with one electron sheet but also many-layered systems can be fabricated $[5,6]$. The distance between these electron sheets can be varied artificially. If the inter-sheet distance is on the order of the characteristic scale, the effect of interaction of these sheets is apparent.

In this section, taking as an example the two-electron-sheets structure, we show that the interaction of these sheets leads not to a simple change of localized modes but gives a splitting to additional acoustical waves characterized by other features.

Consider two identical electron sheets placed in the planes $z= \pm L$. Assume that the distance between them, $2 L$, is much larger than their thickness $d$. Analysis of analogues of the wave equations (13) and boundary conditions (14)-(16) shows that 
there are two types of solutions: symmetrical and antisymmetrical solutions (related to the change $z \rightarrow-z$ ). We give here only the antisymmetrical solutions:

$$
u_{l l}=\left\{\begin{array}{ll}
\mp A \mathrm{e}^{\mp \kappa(z \pm L)} \\
-A \sinh \kappa z / \sinh \kappa L
\end{array} \quad \phi= \begin{cases}\mp B \mathrm{e}^{\mp q(z \pm L)} & |z|>L \\
-B \sinh q z / \sinh q L & -L<z<L\end{cases}\right.
$$

Here the 'upper' signs are for the region $z>L$ while 'lower' signs are for $z<-L$. $\kappa$ is equal to $\sqrt{q^{2}-\omega^{2} / c_{\mathrm{L}}^{2}}$. Similar to the previous case the relationship between the coefficients of the acoustic and electrostatic wave is given by

$$
B=-A b\left[4 \pi e^{2} P(q, T) / \epsilon_{0}\right] /\left[2 q(1+\operatorname{coth} q L)+4 \pi e^{2} P(q, T) / \epsilon_{0}\right] .
$$

So the equation for $\kappa$ has the following form

$$
\begin{aligned}
\kappa(\omega, q)[1+ & \operatorname{coth}(\kappa(\omega, q) L]=R(q) \\
= & {\left[b^{2} P(q, T) /(\lambda+2 \mu)\right]\left\{q^{3}(1+\operatorname{coth} q L)[2 q(1+\operatorname{coth} q L)\right.} \\
& \left.\left.+4 \pi e^{2} P(q, T) / \epsilon_{0}\right]^{-1}\right\} .
\end{aligned}
$$

The symmetrical solutions can be obtained from (30)-(32) by the simple substitutions $\sinh \rightarrow \cosh$ and coth $\rightarrow$ tanh and taking the same sign for coefficients in (30).

The existence of two types of solution corresponds to the splitting into two sorts of localized modes. The symmetrical one, although differing from the single-electronsheet solutions in the magnitude of the splitting, the degree of localization etc, shows the same physical trends. For examplc, as expected from the physical picture, the symmetrical solutions almost coincide with the onc-sheet mode with doubled $P(q, T)$ at $q L, \kappa L \ll 1$. Both of the solutions, i.e. symmetrical and antisymmetrical, reduce to the one-sheet solutions (17)-(20) at $q L, \kappa L \gg 1$.

However, the antisymmetrical modes are considerably different at small $\kappa, q$. This solution splits from the bulk one after a finite value of $q=q_{\mathrm{c}}$. Since $\kappa_{\text {as }}$ is small for $q \sim q_{\mathfrak{c}}$, we can find the equation for $q_{\mathfrak{c}}$ :

$$
\begin{aligned}
1 / L=R\left(q_{\mathrm{c}}\right) & =\left[b^{2} P\left(q_{\mathrm{c}}, T\right) /(\lambda+2 \mu)\right]\left\{q _ { \mathrm { c } } ^ { 3 } ( 1 + \operatorname { c o t h } q _ { \mathrm { c } } L ) \left[2 q_{\mathrm{c}}\left(1+\operatorname{coth} q_{\mathrm{c}} L\right)\right.\right. \\
& \left.\left.+4 \pi e^{2} P\left(q_{\mathrm{c}}, T\right) / \epsilon_{\mathrm{v}}\right]^{-1}\right\}
\end{aligned}
$$

which always has single root. Near $q_{c}$, a dispersion relation for the antisymmetrical modes is

$$
\omega^{2}=c_{\mathrm{L}}^{2} q^{2}\left(1-\left(R^{\prime}\left(q_{\mathrm{c}}\right)^{2} / c_{\mathrm{L}}^{2} q_{\mathrm{c}}^{2}\right)\left(q-q_{\mathrm{c}}\right)^{2}\right) \quad q>q_{\mathrm{c}}
$$

where the prime denotes the derivative with respect to $q$. Analysis of equation (32) shows that the splitting between bulk phonons and antisymmetrical localized modes increases when $q$ increases up to $2 k_{F}$, then the splitting falls down. The antisymmetrical branch is always placed between the bulk phonons and the symmetrical modes. The phonon spectrum for two identical electron sheets is illustrated in figure 2 by using the structure parameters given at previous section. 


\section{Conclusion}

In this paper, we have shown that the electron sheet, in particular a $2 \mathrm{D}$ electron gas, localizes the acoustic modes due to electron-phonon interaction even for uniform lattice characteristics. The localized modes propagate along the sheet and decay away from it. The acoustic waves are accompanied by the charge waves. The splitting of the modes from the bulk phonons increases when the wave vector $q$ increases and reaches a maximum at $q=2 k_{\mathrm{F}}$, then the split modes converge to the bulk phonons with increasing $q$. Additional features of the localized modes arise for the case of several electron sheets.

From the above results it follows that the localization effect can be significant for media with strong electron-phonon interaction, large effective mass of electrons, high concentration and low temperature.

In conclusion, the localization effect of the electron sheet on the acoustical modes can be considerable. It may be investigated by acoustical $\delta$-doped measurements on semiconductors under optical scattering by the electron sheet, etc. The scattering of the localized modes can affect the clectron transport in these materials.

\section{Appendix}

The surface density of the electrons redistributed into the electron sheet can be calculated by treating the induced potential $h(r)$ as a perturbation. The electron concentration can be written in terms of the density operator $\hat{\rho}$ as

$$
n(\boldsymbol{r})=\operatorname{Tr}\left(\delta\left(\boldsymbol{r}-\boldsymbol{r}^{\prime}\right) \hat{\rho}\left(\boldsymbol{r}^{\prime}\right)\right) .
$$

The Heisenberg equation of motion for the density operator is

$$
\mathrm{i} \hbar \partial \hat{\rho} / \partial t=[H, \hat{\rho}]=H \hat{\rho}-\hat{\rho} H
$$

where the Hamiltonian is $H=H_{0}+h(r, t)$. In the absence of the acoustic wave, the electrons are described by the Hamiltonian $H_{0}$ with eigenfunctions $\psi_{n k}(\boldsymbol{r})=\psi_{n}(z) \mathrm{e}^{\mathrm{i} k \cdot r_{\|}}$, where $\psi_{n}(z)$ corresponds to transverse motion of electrons in the electron sheet and eigenvalues $\epsilon_{n k}, n=1,2, \ldots, k=\left(k_{x}, k_{y}\right)$. The induced potential $h(\boldsymbol{r}, t)=h(\boldsymbol{r}) \mathrm{e}^{\mathrm{j} \omega t-\delta t}$ is introduced as a perturbation, where $h(\boldsymbol{r})$ is given by expression (7) and $\delta \rightarrow 0$ at the end. The initial density operator $\hat{\rho}_{0}$ is diagonal and identical with the Fermi distribution function $f_{0}\left(\epsilon_{n k}\right)$. The solution of equation (36) to first order in $h$ can be found in the interaction representation (i.e. any operator $\hat{A}$ is defined by $\left.\hat{A}_{\mathrm{I}}=\mathrm{e}^{\mathrm{i} H_{0} t / \hbar} \hat{A} \mathrm{e}^{-\mathrm{i} H_{0} t / \hbar}\right)$ by inserting the initial value $\rho(-\infty)=\rho_{0}$

$$
\delta \hat{\rho}_{I}=\hat{\rho}_{I}-\hat{\rho}_{0}=-\frac{i}{\hbar} \int_{-\infty}^{t} d t^{\prime}\left(h_{I}\left(r, t^{\prime}\right) H_{0}-H_{0} h_{I}\left(r, t^{\prime}\right)\right) .
$$

To go further we should take into account criterion (5). Due to criterion (5), we consider the case where the thickness of the electron sheet is much less than the characteristic scale of all functions in the $z$-direction. Hence we can consider that $h$ does not change through the electron sheet and does not lead to intersubband transitions, i.e. $\left\langle\psi_{n, k}|h| \psi_{n^{\prime}, k^{\prime}}\right\rangle=0$ for $n \neq n^{\prime}$, which are concluded from our 
proposed model. Then, the change in density of electrons can be found from equation (35) with the use of $h\left(r_{\|}, 0\right)=h_{q} \mathrm{e}^{\mathrm{i} q \cdot r_{\|}}$as

$$
\delta n(\boldsymbol{r})=2 h\left(\boldsymbol{r}_{\|}, z=0, t\right) \sum_{n k}\left|\psi_{n}(z)\right|^{2} \frac{f_{0}\left(\epsilon_{n, k+q}\right)-f_{0}\left(\epsilon_{n, k}\right)}{\epsilon_{n, k+q}-\epsilon_{n, q}-\hbar \omega+\mathrm{i} \delta} .
$$

In this expression the energy of phonons $h \omega$ can be neglected when compared with the electron energies, that corresponds to the adiabatic (static) limit. After integration over $z$ we have the expression for the surface density of electrons given in (10).

\section{References}

[1] Khourdifi E M and Djafari Rouhani B 1989 J. Phys.: Condens. Matter 17543 1989 Surf. Sci. 211/212 361

[2] Wendler L and Grigoryan V G 1988 Surf. Sci. 206203

[3] Sylla B, Dobrzynskj L and Puszkarski H 1989 J. Phys.: Condens. Matter 11247 Sylla B, More M and Dobrzynski L 1989 Surf. Sci. 213588

[4] Cottam M G and Tilley D R 1989 Introduction to Surface and Superlattice Excitations (Cambridge: Cambridge University Press)

[5] Ploog K 1987 J. Cryst. Growth 81304

[6] Ploog K, Hauser M and Fischer A 1988 Appl. Phys. A 45233

[7] Gülseren $O$ and Ciraci S 1991 Semicond. Sci. Technol. 61002

[8] Keyes R W 1967 Solid State Physics vol 20, ed F Seitz and D Turnbull (New York: Academic) pp 37-90

[9] Landau L D and Lifshitz E M 1986 Theory of Elasticity (Oxford: Pergamon)

[10] Bir G L and Picus G E 1974 Symmemy and Sirain Induced Effects in Semiconductors (New York: Wiley)

[11] Pekar S I, Pipa V I and Piskovoii V N 1970 JETP Leth 12338

Kochelap V A and Sokolov V N 1983 Phys. Starus Solidi b 120565

[12] Kittel C 1968 Solid State Physics vol 22, ed F Seitz and D Turnbull (New York: Academic) pp 1-26

[13] Ye Q Y, Zrenner A, Koch F and Ploog K 1989 Semicond. Sci. TechnoL 4500 